\title{
Hipertensão arterial sistêmica em área urbana no sul do Brasil: prevalência e fatores de risco
}

\section{Systemic arterial hypertension in an urban area of southern Brazil: prevalence and risk factors}

\author{
Roberto Xavier Piccini*, Cesar Gomes Victora*
}

\begin{abstract}
PICCINI, R.X. \& Victora, C.G. Hipertensão arterial sistêmica em área urbana no sul do Brasil: prevalência e fatores de risco. Rev. Saúde Pública, 28: 261-7, 1994. O conhecimento da prevalência de hipertensão arterial sistêmica (HAS) e de seus fatores de risco pode ser de grande valor para orientar o planejamento das políticas de saúde. Para identificar a prevalência de HAS, e sua associação com fatores de risco, foi realizado estudo transversal de base populacional na cidade de Pelotas, no sul do Brasil, onde foram examinadas 1.657 pessoas. A prevalência de HAS foi de $19,8 \%$. Os fatores de risco significativamente associados, após controle para fatores de confusão, foram: cor preta, idade avançada, baixa escolaridade, historia paterna e materna de HAS, uso de sal adicional à mesa e obesidade. A classe social, que mostrou forte associação com HAS na análise bivariada, teve seu efeito reduzido na análise multivariada, quando houve ajuste por sexo, cor e idade.
\end{abstract}

Descritores: Hipertensão, epidemiologia. Fatores de risco.

\section{Introdução}

A hipertensão arterial sistêmica (HAS) é um importante problema de saúde no Rio Grande do Sul, onde as doenças cardiovasculares estão entre as principais causas de morte em adultos ${ }^{15}$.

Entre os portadores de HAS prevalecem os hipertensos leves (85\%), para os quais a identificação do problema e o manejo dos fatores de risco modificáveis pode significar a regressão das cifras tensionais em níveis normais, com redução das conseqüuências nocivas.

Os estudos epidemiológicos sobre HAS, têm enfatizado diferentes aspectos como: terapêuti-

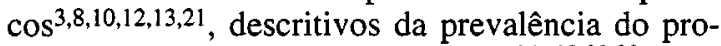
blema e/ou de seus fatores de risco ${ }^{14,19,20,22}$, bem como os relativos à associação de HAS com as doenças cardiovasculares $6,10,18$.

No Estado do Rio Grande do Sul, nos últimos anos, dois estudos, de Costa $^{2}$ (1983) e Ducan ${ }^{4}$ (1991), merecem destaque, sendo que o último ${ }^{4}$ desdobrou em quatro bairros a cidade de Porto Alegre, e a HAS foi abordada como fator de risco para doenças crônico-degenerativas.

\footnotetext{
* Departamento de Medicina Social da Faculdade de Medicina da Universidade Federal de Pelotas - Pelotas, RS - Brasil
}

Separatas/Reprints: R. X. Piccini - Av. Duque de Caxias, 250 96030-000 - Pelotas, RS - Brasil
No presente artigo serão enfocados um estudo epidemiológico transversal de base populacional, realizado na área urbana da cidade de Pelotas, e os respectivos resultados relativos à prevalência de HAS e de alguns de seus fatores de risco, bem como as associações entre estes e HAS.

\section{Metodologia}

O presente estudo foi realizado na área urbana da cidade de Pelotas, na região sul do Brasil (população de 300.000 habitantes), no período de março a junho de 1992.

O delineamento escolhido foi do tipo transversal de base populacional. Assim, foram sorteados 25 setores censitários na área urbana da cidade. Em cada setor foi sorteado um quarteirão e, nele, a esquina para iniciar o estudo. A partir do primeiro domicílio, de forma sistemática, foram visitados um em cada quatro, sempre no sentido da esquerda de quem estivesse de costas para a porta do domicílio inicial, até que fosse completado o total de 36 domicílios por setor. Em cada domicílio todos os adultos de 20 a 69 anos de idade foram entrevistados, pesados, medidos e tiveram sua pressão arterial aferida.

O tamanho da amostra foi dimensionado para detectar uma razão de prevalências igual a 2 para um fator de risco que atingisse $20 \%$ da amostra, com alfa de $5 \%$, poder de $90 \%$ e $15 \%$ de doença nos expostos. Esta foi de 1.390 pessoas ou cerca de 610 domicílios. 
Tal amostra seria também suficiente para estimar uma prevalência de HAS de $15 \%$ com erro inferior a $2 \%$.

Devido ao fato do trabalho de campo ter ocorrido acoplado a outro estudo que requeria uma amostra maior, o número de domicílios foi expandido para 900 (o que resultou em uma amostra substancialmente superior à requerida) tendo este fato aumentado o poder estatístico e a precisão do presente estudo.

Os domicílios onde ninguém estivesse presente e/ou as recusas foram considerados como perdas. $O$ índice de perdas aceitável foi de $10 \%$, sendo que o ocorrido foi de $9,7 \%$, o que resultou em 1.657 indivíduos examinados.

A execução do trabalho de campo ficou a cargo de trinta entrevistadores, estudantes de medicina (segundo ao décimo semestre), todos submetidos a treinamento prévio e participação no estudo piloto.

Ocorreu revisita ao acaso em $5 \%$ dos domicílios, realizada por um segundo entrevistador, no sentido de comprovar que a entrevista havia sido feita e verificar a consistência das informações.

O questionário utilizado para os dados passíveis de coleta por entrevista (sexo, idade, cor, renda, escolaridade, ocupação, história familiar de HAS, padrão de atividade física, tabagismo, consumo de sal e álcool) foi padronizado, précodificado e testado previamente. Balanças de banheiro calibradas foram utilizadas para pesar as pessoas e antropômetros para medi-las.

$O$ indicador para aferir obesidade foi o Índice de Massa Corporal, definido como o peso em kilogramas dividido pela altura em metros ao quadrado. Os pontos de corte para definir obesidade fo- ram os adotados pelo "National Health and Examination Survey II" dos EUA (National Center for Health Statistics, 1978), sendo $27,3 \mathrm{~kg} / \mathrm{m}^{2}$ para mulheres e $27,8 \mathrm{~kg} / \mathrm{m}^{2}$ para homens ${ }^{16}$.

A pressão arterial (PA) foi aferida por esfigmomanômetros aneróides e tomada sempre ao final de cada entrevista (medida única), com a pessoa sentada tendo o braço direito apoiado na altura da região mamária. $\mathrm{O}$ início dos ruídos auscultatórios foi considerado como PA sistólica e a extinção dos ruídos auscultatórios (fase IV), como PA diastólica. Os aparelhos foram revisados semanalmente, sendo utilizado um de mercúrio.

Foram considerados hipertensos aqueles cujas cifras tensionais mostraram valores superiores a $160 \mathrm{mmHg}$ para a PA sistólica e/ou acima de 95 $\mathrm{mmHg}$ para a PA diastólica, ou aqueles que, apesar de apresentarem cifras abaixo das mencionadas, estivessem sob qualquer tratamento farmacológico para HAS.

As classificações de classe social da ABIPEME/IBOPE e a proposta por Bronfman foram utilizadas para medir esta variável ${ }^{1,5,11}$.

O programa Epi Info foi adotado para a entrada dos dados, sendo que a análise foi realizada com os pacotes estatísticos SPSS/PC+ e EGRET.

A medida de efeito usada foi a razão de prevalência e o intervalo de confiança adotado de $95 \%$.

A análise multivariada foi executada por regressão logística não condicional, de acordo com o modelo teórico adotado. Assim, o primeiro nível hierárquico foi composto por sexo, idade e cor, uma vez que estas poderiam afetar todas as demais variáveis. No segundo nível foi acrescentada classe social conforme a classificação de Bronf-

Tabela 1. Fatores de risco (variáveis demográficas) para hipertensão arterial sistêmica. Pelotas, Brasil, 1992.

\begin{tabular}{lcccc}
\hline Variável & $\begin{array}{c}\text { Percentual da } \\
\text { amostra (\%) }\end{array}$ & $\begin{array}{c}\text { Prevalência de } \\
\text { HAS (\%) }\end{array}$ & $\begin{array}{c}\text { Razão de prevalência } \\
\text { (Int. Conf. de 95\%) }\end{array}$ & $P$ \\
\hline $\begin{array}{l}\text { Sexo } \\
\text { Feminino }\end{array}$ & 56,5 & & & \\
Masculino & 43,5 & 21,2 & 1,00 & 0,2 \\
& & 18,3 & $0,86(0,71-1,05)$ & \\
Cor da pele & 81,4 & & & \\
Branca & 18,6 & 18,4 & 1,00 & \\
Preta & & 26,8 & $1,46(1,17-1,81)$ & \\
& & & & \\
Idade em anos & 24,3 & & & \\
estratificada & 28,3 & 2,3 & 1,001 & \\
20-29 anos & 19,4 & 9,0 & $4,01(1,97-8,13)$ & \\
30-39 anos & 17,2 & 27,8 & $12,36(6,33-24,15)$ & \\
$40-49$ anos & 10,8 & 37,1 & $16,49(8,49-32,02)$ & \\
50-59 anos & 46,9 & $20,84(10,72-40,51)$ & \\
60-69 anos & & & & \\
\hline
\end{tabular}

Total de pessoas $=1.657$ 
man. A seguir ingressaram escolaridade e classe social segundo ABIPEME/IBOPE. No quarto e último nível foram introduzidas as demais variáveis.

\section{Resultados}

Entre os 1.657 indivíduos estudados, foram diagnosticados, de acordo com os critérios estabelecidos, 328 hipertensos, o que representa uma prevalência de $19,8 \%$, sendo: 18 pessoas $(5 \%$ dos hipertensos) por hipertensão sistólica, 116 pessoas (36\% dos hipertensos) por hipertensão diastólica, 91 pessoas (27\% dos hipertensos) por hipertensão sistólica e diastólica, e 103 pessoas (32\% dos hipertensos) utilizando medicação anti-hipertensiva com cifras tensionais dentro dos limites da normalidade (HAS compensada).

As Tabelas 1 e 4 mostram variáveis demográficas, familiares, comportamentais e socioeconômicas, respectivamente.

Em cada uma das Tabelas estão dispostos: o percentual da amostra que cada uma das categorias representou, a prevalência de HAS em cada subgrupo, a razão de prevalências e seu intervalo de confiança (95\%) considerando como categoria de base aquela em que se esperava o menor risco e também a significância estatística da associação entre a variável em questão e HAS.

As maiores prevalências de HAS foram detectadas entre pessoas de cor preta. Não foi possível captar diferença significativa entre as prevalências de HAS em ambos os sexos. A ocorrência de HAS foi maior nas pessoas mais idosas (Tabela 1).

O relato positivo de história famillar de HAS, tanto paterna quanto materna, mostrou associação com prevalências significativamente maiores de HAS (Tabela 2).
Entre as variáveis comportamentais, o padrão sedentário de atividade física representou fator de risco positivo e significativamente associado com HAS. O consumo de álcool até duas vezes por semana e tabagismo atual representaram fatores de proteção significativos para HAS (Tabela 3).

Com relação à classe social e escolaridade, as maiores prevalências de HAS foram detectadas nas categorias sociais menos privilegiadas (Tabela 4).

A presença de obesidade em $33 \%$ da amostra constituiu um achado extremamente relevante, sendo que entre os obesos a prevalência de HAS foi de $33 \%$ e a razão de prevalências com relação aos indivíduos de peso corporal normal foi de 2,52 (IC $95 \%$ de 2,08 a 3,05) e $p<0,001$.

A análise multivariada por regressão logística, de acordo com o modelo teórico descrito, mostrou a persistência, como fatores de risco significativamente associados à HAS, das seguintes variáveis: cor preta, idade avançada, obesidade, baixa escolaridade, história paterna e materna de HAS. Através da análise acima citada, apareceu o efeito de risco do uso de sal adicional na mesa e desapareceram os efeitos de proteção do álcool e tabaco (Tabela 5).

\section{Discussão}

O delineamento transversal tem como principais vantagens a rapidez, os custos relativamente baixos e o fato de possuir atributos que permitem, a partir de amostragem representativa de uma população, descrever características da mesma e ainda explorar possíveis associações entre os fatores de risco estudados, desde que esta análise se submeta a um modelo préestabelecido.

Tabela 2. Fatores de risco (variáveis familiares) para hipertensão arterial sistêmica. Pelotas, Brasil, 1992.

\begin{tabular}{lcccc}
\hline Variável & $\begin{array}{c}\text { Percentual da } \\
\text { amostra (\%) }\end{array}$ & $\begin{array}{c}\text { Prevalência de } \\
\text { HAS (\%) }\end{array}$ & $\begin{array}{c}\text { Razão de prevalência } \\
\text { (Int. Conf. de 95\%) }\end{array}$ & $P$ \\
\hline $\begin{array}{l}\text { Histórica } \\
\text { paterna de HAS }\end{array}$ & & & & $<0,001$ \\
Não & 55,4 & 15,0 & 1,00 & \\
Não sabe & 25,3 & 25,5 & $1,70(1,35-2,13)$ & \\
Sim & 19,4 & 26,6 & $1,77(1,40-2,25)$ & \\
História & & & & \\
materna de HAS & & & & \\
Náo & 44,9 & 14,7 & 1,00 & \\
Não sabe & 18,2 & 25,8 & $1,75(1,35 \cdot 2,27)$ & \\
Sím & 36,9 & 23,4 & $1,59(1,27 \cdot 1,99)$ & \\
\hline
\end{tabular}

Total de pessoas $=1.657$ 
Tabela 3. Fatores de risco (variáveis comportamentais) para hipertensão arterial sistêmica. Pelotas, Brasil, 1992.

\begin{tabular}{|c|c|c|c|c|}
\hline Variável & $\begin{array}{l}\text { Percentual da } \\
\text { amostra }(\%)\end{array}$ & $\begin{array}{l}\text { Prevalência de } \\
\text { HAS (\%) }\end{array}$ & $\begin{array}{l}\text { Razăo de prevalência } \\
\text { (Int. Conf. de } 95 \% \text { ) }\end{array}$ & $P$ \\
\hline $\begin{array}{l}\text { Tabagismo } \\
\text { Nunca fumou } \\
\text { Ex-fumante } \\
\text { Fumante atual }\end{array}$ & $\begin{array}{l}46,9 \\
17,9 \\
35,3\end{array}$ & $\begin{array}{l}20,9 \\
25,2 \\
16,0\end{array}$ & $\begin{array}{l}1,00 \\
1,21(0,95-1,53) \\
0,77(0,61 \cdot 0,97)\end{array}$ & $=0,003$ \\
\hline $\begin{array}{l}\text { Uso de álcool } \\
\text { Não } \\
\text { Sim }\end{array}$ & $\begin{array}{l}49,9 \\
50,1\end{array}$ & $\begin{array}{l}22,9 \\
17,0\end{array}$ & $\begin{array}{l}1,00 \\
0,74(0,61-0,90)\end{array}$ & $=0,003$ \\
\hline $\begin{array}{l}\text { Padrão de consumo } \\
\text { alcoólico } \\
\text { Não bebe } \\
1 \text { a } 3 \text { vezes/mês } \\
1 \text { a } 2 \text { vezes/sem. } \\
3 \text { a } 6 \text { vezes/sem. } \\
\text { Diariamente }\end{array}$ & $\begin{array}{r}50,2 \\
19,5 \\
17,5 \\
4,0 \\
8,9\end{array}$ & $\begin{array}{l}23,0 \\
15,9 \\
15,0 \\
13,8 \\
24,0\end{array}$ & $\begin{array}{l}1,00 \\
0,70(0,53 \cdot 0,93) \\
0,66(0,49 \cdot 0,89) \\
0,61(0,33 \cdot 1,13) \\
1,05(0,77 \cdot 1,44)\end{array}$ & $=0,003$ \\
\hline $\begin{array}{l}\text { Sempre adiciona sal na } \\
\text { mesa } \\
\text { Não } \\
\text { Sim }\end{array}$ & $\begin{array}{r}96,4 \\
3,6\end{array}$ & $\begin{array}{l}19,5 \\
26,7\end{array}$ & $\begin{array}{l}1,00 \\
1,36(0,89-2,10)\end{array}$ & $=0,2$ \\
\hline $\begin{array}{l}\text { Ingesta de embutidos } \\
\text { no últimos mês } \\
\text { Não comeu } \\
\text { Comeu }\end{array}$ & $\begin{array}{l}41,9 \\
58,1\end{array}$ & $\begin{array}{l}23,9 \\
17,0\end{array}$ & $\begin{array}{l}1,00 \\
0,71(0,59-0,86)\end{array}$ & $<0,001$ \\
\hline $\begin{array}{l}\text { Comer churrasco no } \\
\text { último mês } \\
\text { Não comeu } \\
\text { Comeu }\end{array}$ & $\begin{array}{l}46,0 \\
54,0\end{array}$ & $\begin{array}{l}21,8 \\
18,3\end{array}$ & $\begin{array}{l}1,00 \\
0,84(0,69-1,02)\end{array}$ & $=0,09$ \\
\hline $\begin{array}{l}\text { Padrão de atividade } \\
\text { física no último ano } \\
\text { Ambos } \\
\text { Só lazer } \\
\text { Só deslocamente } \\
\text { Nenhum }\end{array}$ & $\begin{array}{r}8,4 \\
16,2 \\
19,5 \\
55,8\end{array}$ & $\begin{array}{r}7,9 \\
17,8 \\
15,2 \\
23,8\end{array}$ & $\begin{array}{l}1,00 \\
2,27(1,22-4,23) \\
1,93(1,04 \cdot 3,60) \\
3,03(1,70 \cdot 5,40)\end{array}$ & $<0,001$ \\
\hline
\end{tabular}

Total de pessoas $=1.657$

A grande limitação deste delineamento é a dificuldade de estabelecer causalidade uma vez que, tratando-se de um corte no tempo, os possíveis determinantes e o desfecho são vistos em um mesmo momento, impossibilitando a utilização da temporalidade como critério causal.

As dificuldades metodológicas deste delineamento são o tamanho amostral e a aleatoriedade em sua seleção. Além destas, em estudos transversais de grande porte, os aspectos logísticos da reprodutibilidade e confiabilidade na coleta de dados também constituem dificuldades relevantes.

As dificuldades acima mencionadas foram consideradas com extrema cautela no presente estudo. Entre outras iniciativas foi realizado minucioso treinamento dos entrevistadores e empreendida revisita, por outro entrevistador, em uma sub- amostra de $5 \%$ da amostra principal, também selecionada ao acaso.

Entre os resultados encontrados destacou-se a prevalêncla de $19,8 \%$ de HAS na população de 20 a 69 anos de idade. O estudo de Duncan ${ }^{4}$ mostrou uma prevalência de $15 \%$ em amostra de quatro bairros da cidade de Porto Alegre. Entretanto, a faixa etária da população estudada foi de 15 a 64 anos o que talvez possa justificar a diferença. Já o estudo de Costa ${ }^{2}$ acusou uma prevalência de $12 \%$ em amostra da população de 20 a 74 anos de idade do Estado do Rio Grande do Sul em 1978. Os dados internacionais mostraram valores similares, tais como $15 \%$, citado por Fry ${ }^{7}$ e $20 \%$ mencionado por Hurst ${ }^{9}$.

Outros resultados também dignos de destaque foram as prevalências dos fatores de risco que dependem de hábitos de vida (Tabela 3), todas com 
Tabela 4. Fatores de risco (variáveis socioeconômicas) para hipertensāo arterial sistêmica. Pelotas, Brasil, 1992.

\begin{tabular}{|c|c|c|c|c|}
\hline Variável & $\begin{array}{l}\text { Percentual da } \\
\text { amostra (\%) }\end{array}$ & $\begin{array}{c}\text { Prevalência de } \\
\text { HAS (\%) }\end{array}$ & $\begin{array}{l}\text { Razão de prevalência } \\
\text { (Int. Conf. de } 95 \% \text { ) }\end{array}$ & $P$ \\
\hline $\begin{array}{l}\text { Nivel de escolaridade } \\
\text { completado }\end{array}$ & & & & $<0,001$ \\
\hline $\begin{array}{l}\geq 11 \text { anos } \\
8-10 \text { anos } \\
5-7 \text { anos } \\
1-4 \text { anos } \\
\text { Sem estudo }\end{array}$ & $\begin{array}{r}25,7 \\
18,5 \\
27,9 \\
19,2 \\
8,8\end{array}$ & $\begin{array}{l}10,6 \\
10,8 \\
19,5 \\
29,9 \\
44,8\end{array}$ & $\begin{array}{l}1,00 \\
1,02(0,67-1,56) \\
1,84(1,32-2,57) \\
2,83(2,05-3,91) \\
4,24(3,05-5,90)\end{array}$ & \\
\hline $\begin{array}{l}\text { Classe social } \\
\text { Burg. / Nov. pq. burg. } \\
\text { Peq. burg. trad. } \\
\text { Prolet. não típico } \\
\text { Prolet. típico } \\
\text { Subproletariado } \\
\text { Fora da população } \\
\text { economicamente ativa }\end{array}$ & $\begin{array}{r}10,2 \\
20,2 \\
44,5 \\
13,9 \\
7,4 \\
3,7\end{array}$ & $\begin{array}{l}11,8 \\
18,0 \\
20,2 \\
23,8 \\
22,8 \\
25,8\end{array}$ & $\begin{array}{l}1,00 \\
1,52(0,95-2,43) \\
1,71(1,10-2,64) \\
2,01(1,26 \cdot 3,22) \\
1,92(1,14-3,25) \\
2,18(1,21-3,93)\end{array}$ & $=0,03$ \\
\hline $\begin{array}{l}\text { Classe social segundo } \\
\text { ABIPEME/IBOPE1,5.11 } \\
\text { Classe A/B } \\
\text { Classe C } \\
\text { Classe D } \\
\text { Classe E }\end{array}$ & $\begin{array}{l}10,3 \\
23,2 \\
35,5 \\
31,0\end{array}$ & $\begin{array}{l}14,0 \\
15,6 \\
21,4 \\
23,0\end{array}$ & $\begin{array}{l}1,00 \\
1,11(0,72-1,72) \\
1,53(1,02-2,28) \\
1,64(1,09-2,45)\end{array}$ & $=0,006$ \\
\hline
\end{tabular}

Total de pessoas $=1.657$

Tabela 5. Análise multivariada por regressão logística hierarquizada de fatores de risco para hipertensão arterial sistêmica. Pelotas, Brasil, 1992.

\begin{tabular}{|c|c|c|}
\hline Variável & Odds Ratio (1.C. $95 \%$ ) & $\mathrm{P}$ \\
\hline $\begin{array}{l}\text { Sexo } \\
\text { Feminino } \\
\text { Masculino }\end{array}$ & $\begin{array}{l}1,00 \\
0,77(0,58-1,01)\end{array}$ & $=0,06$ \\
\hline $\begin{array}{l}\text { Idade* } \\
20-29 \text { anos } \\
30.39 \text { anos } \\
40.49 \text { anos } \\
50-59 \text { anos } \\
60-69 \text { anos }\end{array}$ & $\begin{array}{c}1,00 \\
4,49(2,15-9,37) \\
17,44(8,59-35,38) \\
28,32(13,94-57,53) \\
42,00(20,26-87,08)\end{array}$ & $<0,001$ \\
\hline $\begin{array}{l}\text { Cor* } \\
\text { Branca } \\
\text { Preta }\end{array}$ & $\begin{array}{l}1,00 \\
2,11(1,52-2,92)\end{array}$ & $<0,001$ \\
\hline $\begin{array}{l}\text { Classe social** } \\
\text { Burg./Nov. Peq. Burg. } \\
\text { Peq. Burg. Trad. } \\
\text { Prolet. não típico } \\
\text { Prolet. tipico } \\
\text { Subproletariado } \\
\text { Fora da população } \\
\text { economicamente ativa }\end{array}$ & $\begin{array}{l}1,00 \\
1,43(0,80-2,56) \\
1,77(1,04-3,03) \\
2,09(1,14-3,83) \\
1,45(0,72 \cdot 2,91) \\
1,36(0,60-3,07)\end{array}$ & \\
\hline $\begin{array}{l}\text { Nivel de escolaridade } \\
\text { completado** } \\
\geq 11 \text { anos } \\
8-10 \text { anos } \\
5-7 \text { anos } \\
1-4 \text { anos } \\
\text { Sem estudo }\end{array}$ & $\begin{array}{l}1,00 \\
0,88(0,52-1,49) \\
1,36(0,87-2,14) \\
1,60(1,00-2,55) \\
2,31(1,34-3,96)\end{array}$ & $=0,004$ \\
\hline
\end{tabular}

Continuação da tabela 5.

\begin{tabular}{|c|c|c|}
\hline Variável & Odds Ratio (l C 95\%) & $P$ \\
\hline $\begin{array}{l}\text { Classe social segundo } \\
\text { ABIPEME/IBOPE*** } \\
\text { Classe A/B } \\
\text { Classe C } \\
\text { Classe D } \\
\text { Classe E }\end{array}$ & $\begin{array}{l}1,00 \\
0,95(0,50-1,82) \\
1,13(0,58-2,22) \\
0,92(0,45-1,90)\end{array}$ & $=0,6$ \\
\hline $\begin{array}{l}\text { Indice de massa } \\
\text { corporal (Quetelet) } \\
\text { Normal } \\
\text { Obeso }\end{array}$ & $\begin{array}{l}1,00 \\
2,03(1,52-2,71)\end{array}$ & $<0,001$ \\
\hline $\begin{array}{l}\text { Uso de álcool****} \\
\text { Não } \\
\text { Sim }\end{array}$ & $\begin{array}{l}1,00 \\
1,08(0,80-1,45)\end{array}$ & $=0,6$ \\
\hline $\begin{array}{l}\text { Padrão de atividade } \\
\text { física no último ano**** } \\
\text { Ambos } \\
\text { Só lazer } \\
\text { Só deslocamento } \\
\text { Nenhum }\end{array}$ & $\begin{array}{l}1,00 \\
1,07(0,49-2,30) \\
1,51(0,70-3,22) \\
1,41(0,69-2,89)\end{array}$ & $=0,4$ \\
\hline $\begin{array}{l}\text { Tabagismo } \\
\text { Nunca fumou } \\
\text { Ex-fumante } \\
\text { Fumante atual }\end{array}$ & $\begin{array}{l}1,00 \\
1,17(0,80-1,71) \\
0,92(0,65-1,29)\end{array}$ & $=0,5$ \\
\hline $\begin{array}{l}\text { Sempre adiciona sal na } \\
\text { mesa**** } \\
\text { Não } \\
\text { Sim }\end{array}$ & $\begin{array}{l}1,00 \\
2,41(1,18-0,91)\end{array}$ & $=0,02$ \\
\hline
\end{tabular}


Continuação da tabela 5 .

\begin{tabular}{llc}
\hline Variável & Odds Ratio (I C 95\%) & $P$ \\
\hline $\begin{array}{l}\text { Comer churrasco no } \\
\text { último mês**** }\end{array}$ & & $=0,7$ \\
Não & 1,00 & \\
Sim & $1,06(0,78-1,45)$ & \\
& & \\
Ingesta de embutidos & & \\
no último mês**** & 1,00 & \\
Não & $0,90(0,67-1,21)$ & \\
Sim & & \\
História familiar & & \\
paterna de HAS & & \\
Não & 1,00 & \\
Não sabe & $1,20(0,86-1,67)$ & \\
Sim & $2,16(1,51-3,08)$ & \\
História familiar & & \\
materna de HAS & & \\
Não & & \\
Não sabe & 1,00 & \\
Sim & $0,97(0,58-1,61)$ & \\
\hline
\end{tabular}

Total de pessoas $=1.657$

* Modelo 1: sexo, idade e cor.

* Modelo 2: modelo (1) mais classe social.

*** Modelo 3: Modelo (2) mais escolaridade e classe social segundo ABIPEME/IBOPE.

**** Modelo 4: modelo (3) acrescido dos demais fatores de risco.

valores acima de $35 \%$, exceto o uso de sal adicional na mesa. Estes dados sugeriram a necessidade de planejamento com vistas a intervenção eficaz e eficiente em sua abordagem.

$\mathrm{Na}$ análise bivariada, com exceção de sexo e ingesta de churrasco, todas as variáveis estudadas mostraram alguma associação com HAS. Entretanto, após o controle para variáveis de confusão, através de regressão logística hierarquizada, permaneceram como fatores de risco apenas: idade, cor, escolaridade, obesidade e história familiar, aparecendo o efeito do uso de sal adicional na mesa. Como a prevalência deste uso foi baixa e os demais fatores de risco estudados apresentavam perspectiva de abordagem inviável ou muito difícil, a obesidade assumiu papel de destaque no modelo teórico de determinação da HAS em termos de estratégias de prevenção.

O efeito global da variável classe social não foi significativo na análise multivariada. No entanto, duas categorias, o proletariado típico e o proletariado não-típico, permaneceram com risco significativamente aumentado, o que ficou evidenciado pelo limite inferior do intervalo de confiança maior que a unidade.

$\mathrm{O}$ aparente efeito protetor da categoria de fumante atual desapareceu na análise multivariada, não tendo assumido tampouco o papel de risco, o que mostrou concordância com os dados da literatura ${ }^{17}$.

Os resultados mostraram HAS como problema prevalente na população adulta da cidade de Pelotas. As variáveis sociais representaram um papel destacado no modelo de determinação da HAS adotado, assim como a informação positiva de história familiar. A obesidade foi fator de risco digno de especial atenção por sua condição de elevada prevalência, bem como pela possibilidade de modificação desta através de intervenção adequadamente planejada.

PICCINI, R. X. \& VICTORA, C. G. [Systemic arterial hypertension in an urban area of southern Brazil: prevalence and risk factors]. Rev. sSúde Pública, 28: 261-7, 1994. Knowledge of the prevalence of systemic arterial hypertension and its risk factors can be of great value to health policy and planning activities. A cross-sectional study was carried out in Pelotas, southern Brazil, for the purpose of discovering the prevalence of hypertension and selected risk factors. A representative sample of 1,675 adults were studied. The prevalence of hypertension was of $19.8 \%$. The following variables were significantly associated with hypertension after adjustment for confounding variables: black race, advanced age, low educational level, paternal and maternal history of hypertension, use of additional salt on cooked foods, and obesity. The strong association between social class and hypertension found by bivariant analysis was reduced in the multivariate analysis after adjustment for age, sex and race.

Keywords: Hypertension, epidemiology. Risk factors.

\section{Referências Bibliográficas}

1. BRONFMAN, M. \& TUIRÁN, R.A. La desigualdad social ante la muerte: clases sociales y mortalidad en la niñez. Cuad. Med. Soc., (29/30): 198-4, 1985.

2. COSTA, E.A. Hipertensão como problema de massa no Brasil: caracteres epidemiológicos e fatores de risco. Ciên. e Cull., 35: 1642-9, 1983.

3. CRUICKSHANK, J.N. et al. Benefits and potential harm of lowering high blood pressure. Lancet, 1:581-3, 1987.

4. DUNCAN, B.B. As desigualdades sociais na distribuição de fatores de risco para doenças não transmissíveis. Porto Alegre, 1991. [Tese de Doutorado - Faculdade de Medicina/UFRGS].

5. EDITORIAL. Isto é Senhor (1158): 24-5, 1991.

6. EUROPEAN WORKING PARTY OF HIGH BLOOD PRESSURE IN THE ELDERLY. Mortality and morbidity results from the EWPHP. Lancet, 1: 1349-54, 1985.

7. FRY, J. Doenças comuns: incidência, natureza e tratamento. São Paulo, Editora Manole, 1977.

8. GILL, J.S. et al. Relation betwen initial blood pressure and its fall with treatment. Lancet, 1: 567-9, 1985.

9. HURST, J.W. The heart. Blakinston, 1978. 
10.HYPERTENSION DETECTION AND FOLLOW-UP PROGRAM COOPERATIVE GROUP. The effect of treatment on mortality in mild hypertension. $N$. Engl. J. Med., 307: 976-80, 1982.

11. LOMBARDI, C. et al. Operacionalização do conceito de classe social em estudos epidemiológicos. Rev. Saúde Pública, 22: 253-65, 1988.

12. MANAGEMENT COMMITTEE. The Australian therapeutic trial in mild hypertension. Lancet, 1: 1261-7, 1980.

13. MEDICAL RESEARCH COUNCIL WORKING PARTY. MRC trial of treatment of mild hypertension: principal results. $B M J, 291$ : 97-104, 1985.

14. MILLAR, W.J. \& STEPHENS, T. The prevalence of overweigth and obesity in Britain Canada and United States. Am. J. Public Health, 77: 38-41, 1987.

15. MINISTÉRIO DA SAỨDE. Fundação Nacional de Saúde. Centro Nacional de Epidemiologia. Dez principais causas de óbitos por região, Brasil -1977 a 1988. Inf. Epidemiol. SUS, 1 (4): $91-142,1992$.
16. NAJAR, M.F. \& ROWLAND, M. Anthropometric reference data and prevalence of overweigth, United States, 1976-80. Vital Health Stat., 11 (238):73, 1987.

17. PEACH, H. \& HELLER, R.F. Epidemiology of common diseases. London, William Heinemann Medical Books, 1984.

18. RICHARD, J.L. The epidemiology of coronary heart disease: a review. Effect. Health Care, 2: 197-208, 1985.

19. SACKS, E.M. et al. Blood pressure in vegetarians. Am. J. Epidemiol., 100: 390-8, 1974.

20. SAUNDERS, J.B. Alcohol: an important cause of hypertension. $B M J, 294(6579)$ : 1045-6, 1980.

21. TREATMENT of hypertension: the 1985 results. Lancet, 1 : 645-7, 1985.

22. WEINBERGER, M.H. et al. Dietary sodium restriction as adjunctive treatment of hypertension. JAMA, 259(17): $2561-5,1988$.

Recebido para publicaçāo em 8.2.1994 Reapresentado em 31.5.1994 Aprovado para publicação em 7.7.1994 